\title{
Cooperative Communications System With Spatially Random Relays
}

\author{
Hongzheng Wang, Shaodan Ma, Tung-Sang Ng, \\ Department of Electrical and Electronic Engineering \\ The University of Hong Kong, Hong Kong \\ Email: \{hzwang, sdma, tsng\}@eee.hku.hk
}

\begin{abstract}
Cooperative communications is a multiuser framework for wireless network environments. The collaboration among the users can exploit the inherent spatial diversity gains to thereby enhance the system performance significantly. Due to the mobility of users, especially in cellular systems, the exact locations of them are usually unknown a priori and hard/costly to discover. Therefore it is reasonable to model their locations as Bernoulli process. In this work, the uplink of a microcell in a decodeand-forward cooperative cellular system is investigated. The outage probability is derived based on a practical channel model including not only path loss but also shadowing and multipath fading effects. It is shown that considerable performance gains can be obtained.
\end{abstract}

Index Terms-Cooperative communications, Bernoulli process, path loss, shadowing, multipath fading

\section{INTRODUCTION}

Wireless communications usually suffers from several significant impairments. Despite the additive noise, radio signals are generally affected by reflection, diffraction, and scattering mechanisms [1]. On the other hand, the broadcast nature of radio propagation offers opportunity to exploit the inherent spatial diversity gains and thereby to enhance the performance further. Cooperative communications is such a system, utilizing the unique feature of wireless communications, to provide higher quality of service and better communications experience via coordinations among participants [2], [3]. The benefits of cooperative communications have been justified by a large amount of work, e.g., [2]-[5].

In [2], [3], a simple CDMA system with decode-andforward cooperative scheme is proposed, which can considerably increase the system's performance. In [4], Laneman et al. investigate several cooperation methods and show that the system's outage probability can be significantly decreased by exploiting the so-called cooperative diversity. Most of the literature, especially the work on three-node relay model, focuses on a fixed network topology. It means that the locations of users are exactly known. In practice, however, the users' locations may not be perfectly known due to limited localization accuracy and the mobility of users, especially in cellular systems. Therefore, the randomness of the users should be considered in system design. In [6], a distributed antenna system for the downlink of a cellular system is investigated. The antenna elements are assumed to be randomly distributed and the outage probability in several configurations is analyzed. The links from the base station to the antenna elements are assumed to be fiber optics or line-of-sight RF. That is, the links are error-free, which simplifies the analysis.

In this paper, the uplink of a microcell in a cellular system, where a particular user would like to communicate with the base station through the assistances of other idle users, is considered. Due to the randomness of users, the locations of them are modeled as a Bernoulli process. Unlike the model in [6], no error-free links are assumed in our model, which is usually the case in cellular systems. In addition, to be practical, the channel in this paper considers not only path loss, but also shadowing and multipath fading. Based on the model, the effects of random locations of users on the system performance is analyzed and the outage probability is derived.

\section{System MODEL}

\section{A. System Description}

In this paper, we consider the uplink of a microcell in a cellular system, shown in Fig. 1, where the base station is located at the center and denoted as "destination", and a particular user who has information to transmit is called "source". The cell size is $R_{S}$ and the distance from the source to the destination is $L$. The idle users who may offer cooperations in the cell are represented by other points in the figure, and the total number of idle users is $n$. Due to the mobility of these idle users and possible environment restrictions, their exact locations are unknown to the particular user. Without loss of generality, we assume that there is no clear patterns or trends in the density of these users. Consequently, these location-unknown users can be modeled as a uniform point process [7], i.e., a special case of Bernoulli process.

Definition 1 (Bernoulli process [8]): A Bernoulli process on area $A$ with parameters $n$ and probability measure $P(\cdot)$ is a random finite set $\boldsymbol{\Psi} \subset A$ for which the totality of $\boldsymbol{\Psi}$ is $n$, and

$$
\begin{gathered}
\operatorname{Pr}\left(N\left(A_{1}\right)=n_{1}, N\left(A_{2}\right)=n_{2}, \ldots, N\left(A_{k}\right)=n_{k}\right) \\
\quad=\frac{n !}{n_{0} ! n_{1} ! \cdots n_{k} !} P\left(A_{0}\right)^{n_{0}} P\left(A_{1}\right)^{n_{1}} \cdots P\left(A_{k}\right)^{n_{k}}
\end{gathered}
$$

where $A_{0}, A_{1}, \ldots, A_{k}$ are arbitrary disjoint subsets of $A$ with $\bigcup_{i=0}^{k} A_{i}=A . N\left(A_{i}\right)$ denotes the number of points of $\Psi$ falling in $A_{i}$, and $\sum_{i=0}^{k} n_{i}=n$. When the $n$ points are 


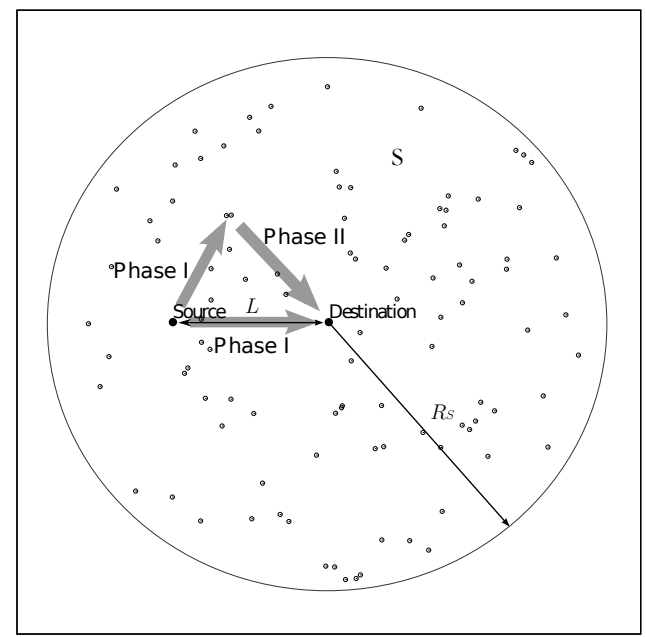

Fig. 1. The uplink of a microcell in a cellular system.

uniformly distributed on $A$, the probability measure $P(\cdot)$ can be simplified as

$$
P\left(A_{i}\right)=\left|A_{i}\right| / \pi R_{S}^{2}
$$

where $\left|A_{i}\right|$ denotes the area of $A_{i}$.

In the uplink system the particular user (the source) intends to transmit information to the base station. In order to increase his/her communications quality, some kind of coordination among these idle users will be utilized and the whole session is divided into two phases: the broadcast phase and the cooperation phase. In the broadcast phase (phase I), the source broadcasts the desired information to the base station (the destination). The received signal at the destination is

$$
d_{1}=h_{s d} \sqrt{P_{s}} s+z_{1},
$$

where $h_{s d}$ is the channel response from the source to the destination, $P_{s}$ is the power utilized by the source, $s$ is the transmitted symbol with unit variance, and $z_{1}$ is the additive white Gaussian noise (AWGN) with power density $N_{0}$. The destination will save copies of the received signal and $h_{s d}$ for later use.

The idle users will also obtain the signal. Similarly, the received signal at an arbitrary idle user is

$$
d_{s r}=h_{s r} \sqrt{P_{s}} s+z_{r},
$$

where $h_{s r}$ is the channel response from the source to the user, and the AWGN $z_{r}$ is also assumed to have power density $N_{0}$. This user may or may not successfully decode the desired symbol depending on whether his/her instantaneous signal-tonoise ratio (SNR) is greater than the threshold $\Theta$ which is a given system parameter. Here the instantaneous SNR $\gamma_{s r}$ is defined as $\left|h_{s r}\right|^{2} P_{s} / N_{0}$. If the user does successfully decode $s$, he/she will be selected as a relay user and will be involved in the second phase. For notational simplicity, we define a set D to represent all these relay users.

In phase II, these relay users from $\mathbf{D}$ decode and forward $s$ to the base station in a cooperative way. The received signal at the destination is

$$
d_{2}=\sum_{i \in \mathbf{D}} h_{r d, i} v_{i} \sqrt{P_{r}} s+z_{2},
$$

where $h_{r d, i}$ is the channel response from $i$ th relay user to the destination, $v_{i}$ is the combination weight, and $P_{r}$ is the power shared by all relay users. The symbol $z_{2}$ represents the AWGN with power density $N_{0}$. Let $P_{\text {tot }}=P_{s}+P_{r}$ be the total power utilized in these two phases. Maximum ratio transmission (MRT) is adopted to achieve the maximum SNR [9], and the weight $v_{i}$ is thus designed as

$$
v_{i}=\frac{h_{r d, i}^{*}}{\sqrt{\sum_{k}\left|h_{r d, k}\right|^{2}}} .
$$

The destination will then constructively combine $d_{1}$ and $d_{2}$ to get the desired information more reliably. Since these two signals are received at different phases, maximum ratio combining (MRC) can be used [10]. It follows that the combined received signal at the destination is

$$
d=g_{1} d_{1}+g_{2} d_{2}
$$

where

$$
\left\{\begin{array}{l}
g_{1}=\frac{h_{s d}^{*} \sqrt{P_{s}}}{\sqrt{\left|h_{s d}\right|^{2} P_{s}+\sum\left|h_{r d, i}\right|^{2} P_{r}}} \\
g_{2}=\frac{\sqrt{\sum\left|h_{r d, i}\right|^{2} P_{r}}}{\sqrt{\left|h_{s d}\right|^{2} P_{s}+\sum\left|h_{r d, i}\right|^{2} P_{r}}} .
\end{array}\right.
$$

After combination, the final SNR $\gamma_{d}$ at the destination is

$$
\gamma_{d}=\gamma_{s d}+\sum_{i \in \mathbf{D}} \gamma_{r d, i}=\frac{P_{s}\left|h_{s d}\right|^{2}}{N_{0}}+\sum_{i=1}^{K} \frac{P_{r}\left|h_{r d, i}\right|^{2}}{N_{0}}
$$

where $K=|\mathbf{D}|, \gamma_{s d}$ is the SNR at the destination contributed by the source in phase I, and $\gamma_{r d, i}$ is the SNR at the destination contributed by $i$ th relay user in phase II.

\section{B. Channel Model}

To be practical, the channel model here includes both path loss, slow shadowing, and fast multipath fading effects. Path loss $\overline{\mathrm{PL}}$ describes the attenuation of the RF signal and gives an area-mean of signal power, which is a decreasing function of distance $r$, i.e., $\overline{\mathrm{PL}}\left(P_{0}, r\right)=P_{0} / N_{0} r^{-\alpha}$ and $\alpha$ is the path loss exponent. Slow shadowing introduces additional fluctuation of signal strength around $\overline{\mathrm{PL}}$, which is a local mean of signal power and is modeled as a log-normal RV $\omega$. Given both path loss and shadowing, the instantaneous signal envelope also suffers fast multipath fading effect, which is modeled as Rayleigh fading here, corresponding to the combination of a large number of independent paths. Based on the channel model, at the end of phase I, the instantaneous SNR $\gamma_{s r}$ at an arbitrary idle user, conditioned on the distance $\rho$ from the source to this user, follows the composite exponential lognormal distribution [6], i.e.

$$
\begin{aligned}
& p_{\gamma_{s r} \mid \rho}(\gamma)=\int_{0}^{\infty} \frac{1}{\omega} \exp \left(-\frac{\gamma}{\omega}\right) \frac{\xi}{\sqrt{2 \pi} \sigma \omega} \\
& \exp \left(-\frac{\left(10 \log _{10} \omega-10 \log _{10} \overline{\mathrm{PL}}\left(P_{s}, \rho\right)\right)^{2}}{2 \sigma^{2}}\right) d \omega
\end{aligned}
$$


where $\xi=10 / \ln 10$, and $\sigma$ is the standard deviation for the log-normal shadowing in $\mathrm{dB}$ and assumed to be constant. Given the distance $l_{i}$ from the $i$ th relay user to the destination, the instantaneous SNR $\gamma_{r d, i}$ has a similar conditional probability distribution

$$
\begin{aligned}
& p_{\gamma_{r d, i} \mid l_{i}}(\gamma)=\int_{0}^{\infty} \frac{1}{\omega} \exp \left(-\frac{\gamma}{\omega}\right) \frac{\xi}{\sqrt{2 \pi} \sigma \omega} \\
& \exp \left(-\frac{\left(10 \log _{10} \omega-10 \log _{10} \overline{\mathrm{PL}}\left(P_{r}, l_{i}\right)\right)^{2}}{2 \sigma^{2}}\right) d \omega
\end{aligned}
$$

The instantaneous SNR $\gamma_{s d}$ obeys the following distribution

$$
\begin{aligned}
& p_{\gamma_{s d}}(\gamma)=\int_{0}^{\infty} \frac{1}{\omega} \exp \left(-\frac{\gamma}{\omega}\right) \frac{\xi}{\sqrt{2 \pi} \sigma \omega} \\
& \exp \left(-\frac{\left(10 \log _{10} \omega-10 \log _{10} \overline{\mathrm{PL}}\left(P_{s}, L\right)\right)^{2}}{2 \sigma^{2}}\right) d \omega
\end{aligned}
$$

\section{Outage Probability AnAlysis}

In this section, we derive the outage probability of the system. The outage probability is defined as $P_{\text {out }}=\operatorname{Pr}\left(\gamma_{d}<\Theta\right)$. According to (9), $\gamma_{d}$ is a sum of $\gamma_{s d}$ and $\gamma_{r d, i}$. Since the distribution of $\gamma_{r d, i}$ depends on the location of the $i$ th relay, the statistical information of $l_{i}$, which is the distance from the $i$ th relay to the destination, is needed for the outage probability analysis. It will be discussed first in the following.

\section{A. Distribution of $l_{i}$}

Since different idle user may have different instantaneous SNR depending on his/her location, the selection of relay users is location-dependent. Mathematically, the relay selection procedure is to mark the points of the uniform point process with two values 0 and 1 , and the points marked with 1 represent selected relays. To derive the exact distribution of $l_{i}$, some facts about marked uniform point process need to be stated as follows.

Definition 2 (Location-dependent mark): For each point $X$ of $\boldsymbol{\Psi}$, we associate a random variable $m_{X}$ (the mark of $X$ ) taking values in some space $M$. If the distribution of $m_{X}$ depends on $X$ but not on the other points of $\Psi$, and the $m_{X}$ for different $X$ are independent, the mark $m_{X}$ is called locationdependent.

Given this definition, we can prove the following theorem for uniform Bernoulli process.

Theorem 1 (Marking theorem for uniform point process): Let $\Psi$ be an $n$-point uniform point process on area $A$. With each point $X$ of $\boldsymbol{\Psi}$, we associate a location-dependent mark $m_{X}$ taking values in 0 or 1 . The points with mark $i(i=0$, 1) form a variable-size Bernoulli process with parameter $K_{i}$ and probability measure $P_{i}(\cdot)$, for which

$$
\begin{aligned}
\operatorname{Pr}\left(K_{i}=k\right)= & \left(\begin{array}{l}
n \\
k
\end{array}\right) \operatorname{Pr}\left(m_{X}(x)=i\right)^{k} \\
& \cdot\left(1-\operatorname{Pr}\left(m_{X}(x)=i\right)\right)^{n-k}, \quad k=0,1, \ldots, n
\end{aligned}
$$

and

$$
P_{i}\left(A^{\prime}\right)=\frac{\int_{A^{\prime}} \operatorname{Pr}\left(m_{X}(x)=i \mid x\right) d x}{\int_{A} \operatorname{Pr}\left(m_{X}(x)=i \mid x\right) d x}
$$

where $A^{\prime}$ is an arbitrary measurable sub-area in $A$.

Note that for notational simplicity we use $x$ to represent the position of a point such that the 2-dimensional integration in terms of $(\rho, \theta)$ in polar coordinate system can be expressed by a normal integration in terms of $x$.

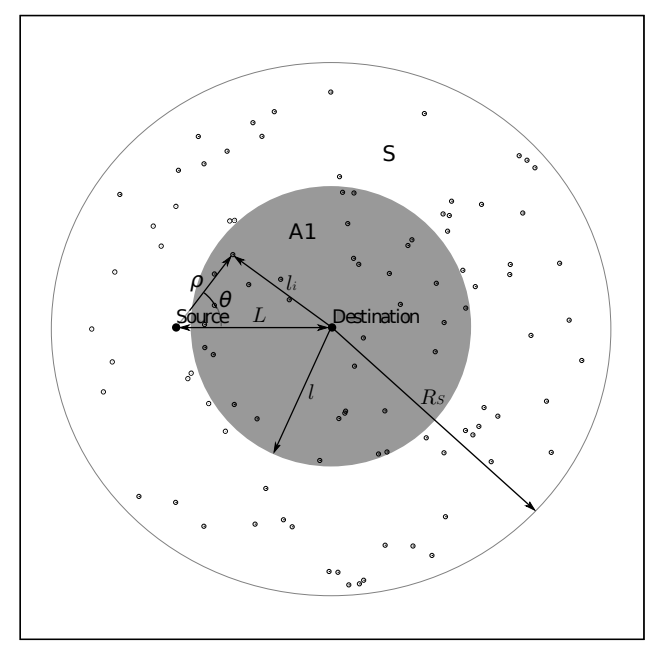

Fig. 2. A pictorial explanation of the distribution of the distance $l_{i}$.

In order to derive the distribution of $l_{i}$, a polar coordinate system with the pole being the source is setup, shown in Fig. 2. Then for an arbitrary idle user located at $(\rho, \theta)$ who is uniformly distributed in $S$, define a binary mark $m_{X}$ satisfying $m_{X}=1$ if $\gamma_{s r}>\Theta$ and $m_{X}=0$ otherwise, i.e.,

$$
\operatorname{Pr}\left(m_{X}=1 \mid X\right)=\operatorname{Pr}\left(\gamma_{s r}>\Theta \mid \rho\right)=\int_{\Theta}^{\infty} p_{\gamma_{s r} \mid \rho}(\gamma) d \gamma,
$$

Then, applying Theorem 1, the probability that a point marked by 1 (relay user) is located in an area $A^{\prime} \in S$ is

$$
\operatorname{Pr}\left(X \in A^{\prime}\right)=\frac{\iint_{A^{\prime}} \rho \operatorname{Pr}\left(\gamma_{s r}>\Theta \mid \rho\right) d \rho d \theta}{\iint_{S} \rho \operatorname{Pr}\left(\gamma_{s r}>\Theta \mid \rho\right) d \rho d \theta} .
$$

Furthermore, the number of relay users $K=|\mathbf{D}|$ is a binomial $\mathrm{RV}$ following the distribution

$$
\begin{gathered}
\operatorname{Pr}(K=k)=\left(\begin{array}{l}
n \\
k
\end{array}\right) \operatorname{Pr}\left(\gamma_{s r}>\Theta\right)^{k}\left(1-\operatorname{Pr}\left(\gamma_{s r}>\Theta\right)\right)^{n-k}, \\
k=0,1, \ldots, n
\end{gathered}
$$

where

$$
\operatorname{Pr}\left(\gamma_{s r}>\Theta\right)=\frac{\int_{0}^{2 \pi} \kappa\left(L \cos \theta+\sqrt{R_{S}^{2}-L^{2} \sin ^{2} \theta}\right) d \theta}{\pi R_{S}^{2}}
$$

and the auxiliary function $\kappa(\cdot)$ is defined as

$$
\kappa(y)=\int_{0}^{y} \rho \operatorname{Pr}\left(\gamma_{s r}>\Theta \mid \rho\right) d \rho=\int_{0}^{y} \rho \int_{\Theta}^{\infty} p_{\gamma_{s r} \mid \rho}(\gamma) d \gamma d \rho .
$$


Let $l_{i}$ denote the distance from the $i$ th relay user to the destination. For any $l \in\left[0, R_{S}\right]$, the probability that $l_{i} \leq l$ is equal to the probability that a relay user is located in the area $A_{1}$, i.e., $\operatorname{Pr}\left(X \in A_{1}\right)$, where $A_{1}$ is the circle area with radius $l$ around the destination, as shown in Fig. 2. Consequently, through (10), (15), and (16), the cumulative distribution function (cdf) of $l_{i}$ can be derived as

$$
P_{l_{i}}(l)=\left\{\begin{array}{c}
\frac{2 \int_{0}^{\theta_{1}} \kappa\left(L \cos \theta+\sqrt{l^{2}-L^{2} \sin ^{2} \theta}\right) d \theta}{\int_{0}^{2 \pi} \kappa\left(L \cos \theta+\sqrt{R_{S}^{2}-L^{2} \sin ^{2} \theta}\right) d \theta} \\
-\frac{2 \int_{0}^{\theta_{1}} \kappa\left(L \cos \theta-\sqrt{l^{2}-L^{2} \sin ^{2} \theta}\right) d \theta}{\int_{0}^{2 \pi} \kappa\left(L \cos \theta+\sqrt{R_{S}^{2}-L^{2} \sin ^{2} \theta}\right) d \theta} \\
0 \leq l<L \\
\frac{\int_{0}^{2 \pi} \kappa\left(L \cos \theta+\sqrt{l^{2}-L^{2} \sin ^{2} \theta}\right) d \theta}{\int_{0}^{2 \pi} \kappa\left(L \cos \theta+\sqrt{R_{S}^{2}-L^{2} \sin ^{2} \theta}\right) d \theta} \\
L \leq l \leq R_{S}
\end{array}\right.
$$

where

$$
\theta_{1}=\arcsin (l / L)
$$

\section{B. Outage Probability}

According to (9), when $K=|\mathbf{D}|=0$, i.e., no relay users are found after phase $\mathrm{I}$, the destination gets only $d_{1}$ through the direct link from the source to the destination. In this case, the pdf of $\gamma_{d}$ is simply given by (12) and can be approximated by the pdf of a single log-normal RV [11]

$$
\begin{aligned}
& p_{\gamma_{d} \mid K=0}(\gamma)=p_{\gamma_{s d}}(\gamma) \approx \\
& \frac{\xi}{\sqrt{2 \pi} \sigma_{s} \gamma} \exp \left(-\frac{\left(10 \log _{10} \gamma-\mu_{s}\right)^{2}}{2 \sigma_{s}^{2}}\right),
\end{aligned}
$$

with

$$
\left\{\begin{array}{l}
\mu_{s}=10 \log _{10} \frac{P_{s}}{N_{0}}-10 \alpha \log _{10} L-\xi \Upsilon \\
\sigma_{s}^{2}=\sigma^{2}+\xi^{2} \frac{\pi^{2}}{6} .
\end{array}\right.
$$

$\Upsilon$ is the Euler's constant [12].

When $K>0$, i.e., at least one relay is found, with the distribution of $l_{i}$ in (20), the pdf of $\gamma_{r d, i}$ is

$$
p_{\gamma_{r d, i}}(\gamma)=\int_{0}^{R_{S}} p_{\gamma_{r d, i} \mid l}(\gamma) d P_{l_{i}}(l)
$$

The complicated expressions of $p_{\gamma_{r d, i} \mid l}$ and $P_{l_{i}}$ make it difficult to obtain analytical results, and motivate us to apply approximations. We propose to approximate $\gamma_{r d, i}$ as a single log-normal RV with pdf being

$$
p_{\gamma_{r d, i}}(\gamma) \approx \frac{\xi}{\sqrt{2 \pi} \sigma_{r} \gamma} \exp \left(-\frac{\left(10 \log _{10} \gamma-\mu_{r}\right)^{2}}{2 \sigma_{r}^{2}}\right),
$$

with

$$
\left\{\begin{array}{l}
\mu_{r}=10 \log _{10} \frac{P_{r}}{N_{0}}-10 \alpha \mathbb{E}\left[\log _{10} l\right]-\xi \Upsilon \\
\sigma_{r}^{2}=\sigma^{2}+\xi^{2} \frac{\pi^{2}}{6}+100 \alpha^{2} \mathbb{D}\left[\log _{10} l\right] .
\end{array}\right.
$$

Given system parameters, e.g., $L, \Theta$ etc, $\mathbb{E}\left[\log _{10} l\right]$ and $\mathbb{D}\left[\log _{10} l\right]$ are constant numbers that can be calculated numerically. The approximation is accurate which can be justified by plotting their cdf curves, i.e., the strategy used in [11].
Consequently, given $K>0, \gamma_{d}$ becomes a sum of lognormal RVs. The distribution of $\gamma_{d}$ has no close-form expression, but can be fairly approximated by a single log-normal distribution with pdf being

$$
\begin{aligned}
& p_{\gamma_{d} \mid K}(\gamma)= \\
& \frac{\xi}{\sqrt{2 \pi} \sigma_{d}(K) \gamma} \exp \left(-\frac{\left(10 \log _{10} \gamma-\mu_{d}(K)\right)^{2}}{2 \sigma_{d}^{2}(K)}\right)
\end{aligned}
$$

There are several methods to calculate the parameters $\mu_{d}(K)$ and $\sigma_{d}(K)$ [13]-[16]. The approach given in [16] is adopted here since it has the best accuracy. The basic idea is to match the moment generating function (MGF) of $\gamma_{d}$ with the MGF of the approximated single log-normal RV with parameters $\mu_{d}(K)$ and $\sigma_{d}(K)$, so that two independent equations can be setup as follows

$$
\begin{aligned}
& \widehat{\Psi}\left(t_{i} ; \mu_{d}(K), \sigma_{d}(K)\right)= \\
& \widehat{\Psi}^{K}\left(t_{i} ; \mu_{r}, \sigma_{r}\right) \cdot \widehat{\Psi}\left(t_{i} ; \mu_{s}, \sigma_{s}\right), \quad i=1,2
\end{aligned}
$$

where

$$
\widehat{\Psi}\left(t ; \mu_{x}, \sigma_{x}\right)=\sum_{n=1}^{N} \frac{w_{n}}{\sqrt{\pi}}\left[-t \exp \left(\frac{\sqrt{2} \sigma_{x} a_{n}+\mu_{x}}{\xi}\right)\right]
$$

is the Gauss-Hermite representation of MGF of log-normal pdf [16]. The weights $w_{n}$ and abscissas $a_{n}$ are tabulated in [12], and $N=16$ is used. The $t_{1}$ and $t_{2}$ are two arbitrarily selected different, real and positive values to make these two equations independent. The parameters $\mu_{d}(K)$ and $\sigma_{d}(K)$ can then be calculated by solving these equations.

Combining the cases of $K=0$ and $K>0$, the pdf of $\gamma_{d}$ can be finally derived as

$$
\begin{aligned}
p_{\gamma_{d}}(\gamma)= & \sum_{k=1}^{n} p_{\gamma_{d} \mid K}(\gamma) \operatorname{Pr}(K=k)+p_{\gamma_{s d}}(\gamma) \operatorname{Pr}(K=0) \\
= & \sum_{k=1}^{n} \frac{\xi}{\sqrt{2 \pi} \sigma_{d}(k) \gamma} \exp \left(-\frac{\left(10 \log _{10} \gamma-\mu_{d}(k)\right)^{2}}{2 \sigma_{d}^{2}(k)}\right) \\
& \cdot\left(\begin{array}{l}
n \\
k
\end{array}\right) \operatorname{Pr}\left(\gamma_{s r}>\Theta\right)^{k}\left(1-\operatorname{Pr}\left(\gamma_{s r}>\Theta\right)\right)^{n-k} \\
& +\frac{\xi}{\sqrt{2 \pi} \sigma_{s} \gamma} \exp \left(-\frac{\left(10 \log _{10} \gamma-\mu_{s}\right)^{2}}{2 \sigma_{s}}\right) \\
& \cdot\left(1-\operatorname{Pr}\left(\gamma_{s r}>\Theta\right)\right)^{n},
\end{aligned}
$$

where $\operatorname{Pr}\left(\gamma_{s r}>\Theta\right)$ is given by (18).

The outage probability is

$$
\begin{aligned}
P_{\text {out }}= & \int_{0}^{\Theta} p_{\gamma_{d}}(\gamma) d \gamma \\
= & \frac{1}{2}+\frac{1}{2} \sum_{k=1}^{n} \operatorname{erf}\left(\frac{10 \log _{10} \Theta-\mu_{d}(k)}{\sqrt{2} \sigma_{d}(k)}\right) \\
& \cdot\left(\begin{array}{l}
n \\
k
\end{array}\right) \operatorname{Pr}(\gamma>\Theta)^{k}(1-\operatorname{Pr}(\gamma>\Theta))^{n-k} \\
& +\frac{1}{2} \operatorname{erf}\left(\frac{10 \log _{10} \Theta-\mu_{s}}{\sqrt{2} \sigma_{s}}\right) \\
& \cdot(1-\operatorname{Pr}(\gamma>\Theta))^{n} .
\end{aligned}
$$




\section{Numerical AND Simulation Results}

In the following simulations, the total power $P_{\text {tot }}$ is set to be $40 \mathrm{~dB}$, and $R_{S}$ is selected so that the edge $\mathrm{SNR}$ is $0 \mathrm{~dB}$. The threshold $\Theta$ is $5 \mathrm{~dB}$. The power allocation factor $\beta$ is defined as the ratio of $P_{s}$ to $P_{\mathrm{tot}}$. Notice that in order to calculate the parameters $\mu_{d}(K)$ and $\sigma_{d}(K)$, the Fenton-Wilkinson (F$\mathrm{W})$ method [13] is first used to generate rough estimation, which are then used as the initial values to solve the nonlinear equations in (28). The path loss exponent $\alpha$ is 4 , and standard deviation for the log-normal shadowing $\sigma$ is $8 \mathrm{~dB}$.

Fig. 3 plots the outage probability of the system as the function of power allocation factor $\beta$ with different number of users $n$. It can be seen that the simulation results and the analysis results are well matched. In addition, the optimal power allocation factor is between 0.6 to 0.8 . Furthermore, when the power allocation factor is 1.0 , the system becomes the conventional non-cooperative system. It shows that the outage probability increases significantly, which justifies the adoption of cooperation among mobile users in cellular systems.

Fig. 4 shows how the normalized distance $L / R_{S}$ affects the system's performance in the terms of outage probability. Obviously, the system will have better performance as the user is getting closer to the destination.

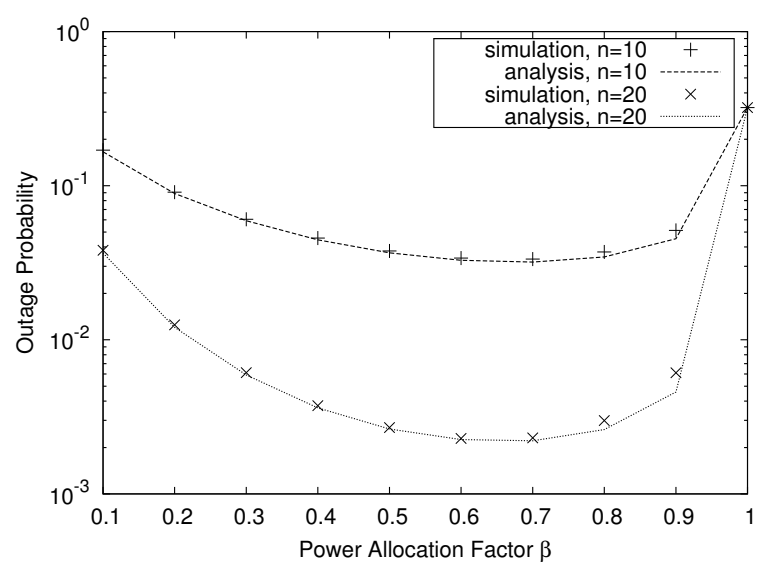

Fig. 3. Power allocation for different $n . \Theta$ is $5 \mathrm{~dB} . L / R_{S}$ is 0.5 . $P_{\text {tot }}$ is $40 \mathrm{~dB}$.

\section{CONCLUSION}

The uplink of a microcell in a cellular system, containing a number of location-random users acting as relays, has been investigated. The outage probability has been derived and verified by the simulations. It has been shown that, with a decode-and-forward cooperative strategy, a particular user can obtain considerable performance gains and has better communications experience than conventional non-cooperative ways. Furthermore, the optimal power allocation scheme has been provided through numerical computations and verified by simulations.

\section{ACKNOWLEDGMENT}

This work was supported by the Hong Kong Research Grants Council (Grant No.: 7154/08E).

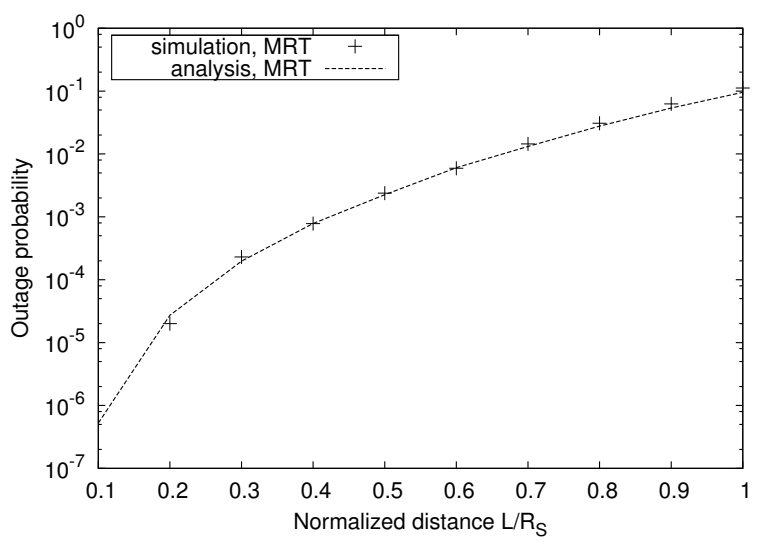

Fig. 4. Outage probability as a function of normalized distance $L / R_{S}$. $P_{\text {tot }}$ is $40 \mathrm{~dB}$. Power allocation factor is 0.7. $n=20 . \Theta$ is $5 \mathrm{~dB}$.

\section{REFERENCES}

[1] G. L. Stüber, Principles of Mobile Communication, 2nd ed. Kluwer Academic Publishers, 2001.

[2] A. Sendonaris, E. Erkip, and B. Aazhang, "User cooperation diversity part I: System description," IEEE Trans. Commun., vol. 51, no. 11, pp. 1927-1938, Nov. 2003.

[3] _ _ "User cooperation diversity - part II: Implementation aspects and performance analysis," IEEE Trans. Commun., vol. 51, pp. 1939-1948, Nov. 2003.

[4] J. N. Laneman, D. N. C. Tse, and G. W. Wornell, "Cooperative diversity in wireless networks: Efficient protocols and outage behavior," IEEE Trans. Inf. Theory, vol. 50, no. 12, pp. 3062-3080, Dec. 2004.

[5] A. S. Ibrahim, A. K. Sadek, W. Su, and K. J. R. Liu, "Cooperative communications with relay-selection: when to cooperate and whom to cooperate with?" IEEE Trans. Wireless Commun., vol. 7, no. 7, pp. 2814-2827, Jul. 2008

[6] J. Zhang and J. Andrews, "Distributed antenna systems with randomness," IEEE Trans. Wireless Commun., vol. 7, no. 9, pp. 3636-3646, Sep. 2008

[7] P. J. Wan and C. W. Yi, "Coverage by randomly deployed wireless sensor networks," IEEE Trans. Inf. Theory, vol. 52, no. 6, pp. 2658-2669, Jun. 2006.

[8] J. F. C. Kingman, Poisson processes. Oxford University Press, 1993.

[9] T. K. Y. Lo, "Maximum ratio transmission," IEEE Trans. Commun., vol. 47 , no. 10 , pp. $1458-1461$, Oct. 1999.

[10] J. G. Proakis, Digital communications. McGraw-Hill Higher Education, 2001.

[11] A. M. D. Turkmani, "Probability of error for m-branch macroscopic selectiondiversity," IEE Proceedings I Communications, Speech and Vision, vol. 139, no. 1, pp. 71-78, Feb. 1992.

[12] M. Abramowitz and I. A. Stegun, Handbook of Mathematical Functions with Formulas, Fraphs, and Mathematical Tables, 10th ed. National Bureau of Standards, 1972. [Online]. Available: http: //www.math.sfu.ca/ c cbm/aands/

[13] L. Fenton, "The sum of log-normal probability distributions in scatter transmission systems," Communications Systems, IRE Transactions on, vol. 8, no. 1, pp. 57-67, Mar. 1960.

[14] S. Schwartz and Y. Yeh, "On the distribution function and moments of power sums with log-normal components," Bell System Technical Journal, vol. 61, no. 7, pp. 1441-1462, 1982.

[15] N. C. Beaulieu and Q. Xie, "An optimal lognormal approximation to lognormal sum distributions," IEEE Trans. Veh. Technol., vol. 53, no. 2, pp. 479-489, Mar. 2004.

[16] N. Mehta, J. Wu, A. Molisch, and J. Zhang, "Approximating a sum of random variables with a lognormal," IEEE Trans. Wireless Commun., vol. 6, no. 7, pp. 2690-2699, Jul. 2007. 